\title{
RESIDUALLY FINITE DIMENSIONAL ALGEBRAS AND POLYNOMIAL ALMOST IDENTITIES
}

\author{
MICHAEL LARSEN AND ANER SHALEV
}

\begin{abstract}
Let $A$ be a residually finite dimensional algebra (not necessarily associative) over a field $k$. Suppose first that $k$ is algebraically closed. We show that if $A$ satisfies a homogeneous almost identity $Q$, then $A$ has an ideal of finite codimension satisfying the identity $Q$. Using well known results of Zelmanov, we conclude that, if a residually finite dimensional Lie algebra $L$ over $k$ is almost $d$-Engel, then $L$ has a nilpotent (resp. locally nilpotent) ideal of finite codimension if char $k=0$ (resp. char $k>0$ ).

Next, suppose that $k$ is finite (so $A$ is residually finite). We prove that, if $A$ satisfies a homogeneous probabilistic identity $Q$, then $Q$ is a coset identity of $A$. Moreover, if $Q$ is multilinear, then $Q$ is an identity of some finite index ideal of $A$.

Along the way we show that, if $Q \in k\left\langle x_{1}, \ldots, x_{n}\right\rangle$ has degree $d$, and $A$ is a finite $k$-algebra such that the probability that $Q\left(a_{1}, \ldots, a_{n}\right)=0$ (where $a_{i} \in A$ are randomly chosen) is at least $1-2^{-d}$, then $Q$ is an identity of $A$. This solves a ring-theoretic analogue of a (still open) group-theoretic problem posed by Dixon,
\end{abstract}

\section{INTRODUCTION}

In this paper, we prove three theorems concerning residually finite dimensional algebras $A$ and polynomial identities. The common theme is that if a (non-commutative) homogeneous polynomial $Q$ in $n$ variables vanishes on a large enough subset of $A^{n}$, then it is actually a coset identity, that is, it holds identically on $\left(a_{1}+I\right) \times \cdots \times\left(a_{n}+I\right)$ for some two-sided ideal $I$ of finite codimension in $A$ and some $a_{1}, \ldots, a_{n} \in A$. Under some assumptions we obtain stronger conclusions, namely, that $Q$ is an identity of the ideal $I$.

Let $k$ be an algebraically closed field, $V$ a $k$-vector space, possibly of infinite dimension, and $n$ a positive integer. We recall [LS] that the codimension of a subset $X \subset V^{n}$ is the smallest integer $c$ for which there exists a direct sum decomposition of $k$-vector spaces $V^{n}=V_{1} \oplus V_{2}$, where $V_{2}$ is finite dimensional, and an algebraic set $X_{2}$ of codimension $c$ in $V_{2}$, such that $X \supset V_{1} \times X_{2}$. We say that $X$ is of infinite codimension if no such decomposition exists.

2010 Mathematics Subject Classification. Primary 16R99.

ML was partially supported by NSF grant DMS-1702152. AS was partially supported by ISF grant $686 / 17$ and the Vinik Chair of mathematics which he holds. Both authors were partially supported by BSF grant 2016072. 
Let $A$ be an associative $k$-algebra, possibly non-unital. Each non-commutative polynomial $Q \in k\left\langle x_{1}, \ldots, x_{n}\right\rangle$ defines the evaluation map $e_{Q}: A^{n} \rightarrow A$. We define $\operatorname{cd}_{Q} A$ to be the codimension of $e_{Q}^{-1}(0)$. We say that $Q$ is an almost identity if $\operatorname{cd}_{Q} A<\infty$. If $\operatorname{cd}_{Q} A=0$, or, equivalently, $e_{Q}\left(A^{n}\right)=0$, we say $Q$ is an identity for $A$.

We can likewise consider a Lie (resp. Jordan) algebra $A$ over $k$ and a Lie (resp. Jordan) polynomial $Q$ and define the codimension of the zero set of $Q$ in $A^{n}$ and an almost identity in the analogous way. In fact the same definition applies for an arbitrary algebra $A$, namely a linear space over $k$ with a bilinear map $A \times A \rightarrow A$ as multiplication (possibly but not necessarily satisfying some extra-conditions). In this case the polynomial $Q$ is an element of a free algebra in the respective category. Note that an ideal of $A$ will always mean a two-sided ideal, and $A$ residually finite dimensional means that the intersection of all ideals of $A$ of finite codimension is the zero ideal.

Our first main result is the following:

Theorem 1. Let $A$ be a residually finite dimensional algebra over $k$ and $Q$ a homogeneous polynomial as above. Then the following are equivalent:

(1) The polynomial $Q$ is an almost identity for $A$.

(2) The polynomial $Q$ is an identity for some ideal I of $A$ of finite codimension.

The non-trivial part is that (1) implies (2). The reverse implication follows from the fact that $I^{n}$ is of finite codimension in $A^{n}$.

We now compare the theorem above with the main result of [LS] (see Theorem 1 there and the comments on p. 10). The latter result shows that an associative/Lie/Jordan algebra with an almost identity $Q$ satisfies some identity $P$ (usually different and more complex than $Q$ ). Theorem 1 above holds for all algebras $A$, and the identity satisfied by the ideal $I$ is the original almost identity $Q$.

Our second main result concerns the case that $k$ is a finite field $\mathbb{F}_{q}$ for a prime power $q$. Any residually finite algebra $A$ over $\mathbb{F}_{q}$ is a dense subalgebra of its completion $\bar{A}$, and as an additive group, $\bar{A}$ is profinite. For each $n$, we endow $\bar{A}^{n}$ with its Haar measure and consider the condition on a subset $X \subset A^{n}$ that $\bar{X}$ has non-zero measure. We say that $Q$ is a probabilistic identity if the closure of $e_{Q}^{-1}(0)$ has positive measure.

Theorem 2. Let $A$ be a residually finite dimensional algebra over $k=\mathbb{F}_{q}$ and $Q$ a homogeneous polynomial as above. Then the following are equivalent:

(1) The polynomial $Q$ is a probabilistic identity of $A$.

(2) The polynomial $Q$ is a coset identity of some finite index ideal I of A.

Furthermore, if $Q$ is multilinear, then these conditions are equivalent to

(3) $Q$ is an identity of some finite index ideal I of $A$. 
Again, the non-trivial part is the claim that (1) implies (2).

In [D] Dixon asks whether, for every group-word $w \in F_{n}$ (the free group of rank $n$ ) there exists $\epsilon=\epsilon(w)>0$ such that if $G$ is a finite group, and the word map $w: G^{n} \rightarrow G$ attains the value 1 with probability $\geq 1-\epsilon$, then $w$ is an identity of $G$. In spite of some positive results for special words $w$, Dixon's Problem is still very much open. Here we obtain a general positive solution of an analogous question on finite algebras $A$. The solution is effective in the sense that $\epsilon$ is given explicitly; in fact, if $d$ is the degree of the ambient polynomial map, then $\epsilon=2^{-d}$ will do.

Theorem 3. Let $A$ be any finite-dimensional algebra over a finite field and $e_{Q}: A^{n} \rightarrow A$ a polynomial map associated with a polynomial $Q$ of degree $d$ in $n$ variables in the respective category. If $\frac{\left|e_{Q}^{-1}(0)\right|}{|A|^{n}} \geq 1-2^{-d}$, then $Q$ is identically zero.

For $d \geq 2$ consider the degree $d$ Engel polynomial $E_{d-1}:=[x, y, \ldots, y]$, a left-normed Lie product where $y$ appears $d-1$ times, as an element of the free Lie algebra on $x, y$ over the underlying field $k$. By [MM, 2.1], if $L$ is a finite Lie algebra in which the Engel condition $E_{d-1}=0$ holds with probability greater than $1-2^{-d}$, then $E_{d-1}$ is an identity of $L$. Theorem 3 above extends this for any finite algebra and any polynomial.

\section{Algebras over algebraically Closed FiEldS}

We recall that every subset $S$ of $k^{m}=\mathbb{A}^{m}(k)$ defines the ideal $Z(S)$ of elements in $k\left[x_{1}, \ldots, x_{m}\right]$ which vanish on $S$ and every ideal $I \subset k\left[x_{1}, \ldots, x_{m}\right]$ defines the algebraic set $V(I)$ of common zeroes of $I$. By Hilbert's Nullstellensatz, these two maps give a bijection between algebraic sets in $k^{n}$ and radical ideals $I$. Radical ideals $I$ are in bijective correspondence with reduced closed $k$-subschemes Spec $k\left[x_{1}, \ldots, x_{m}\right] / I$. The bijection between algebraic sets and reduced closed $k$-subschemes is given in one direction by taking the Zariski closure with its reduced closed subscheme structure and in the other by taking $k$-points.

Each linear transformation $T: V \rightarrow W$ of finite dimensional $k$-vector spaces defines a homomorphism of commutative graded $k$-algebras $T^{*}: \operatorname{Sym}^{*} W^{*} \rightarrow$ Sym $^{*} V^{*}$. If $S_{V} \subset V, S_{T} \subset W$, and $T\left(S_{V}\right) \subset S_{W}$, then $T^{*}\left(Z\left(S_{W}\right)\right) \subset Z\left(S_{V}\right)$. Thus, $T$ determines a morphism of affine $k$-schemes

$$
\text { Spec } \operatorname{Sym}^{*} V^{*} / Z\left(S_{V}\right) \rightarrow \operatorname{Spec~Sym} W^{*} / Z\left(S_{W}\right)
$$

which, at the level of $k$-points, gives the restriction of $T$.

We now prove the key proposition:

Proposition 4. If $A$ is an arbitrary algebra over $k, I$ is an ideal of $A$ of finite codimension, and $Q$ is a homogeneous polynomial as above, then

$$
\operatorname{cd}_{Q} A \geq \operatorname{cd}_{Q} I+\operatorname{cd}_{Q} A / I \text {. }
$$


Proof. There is something to check only if $Q$ is an almost identity for $A$. Let $V_{1} \oplus V_{2}$ be a direct sum decomposition of $A^{n}$ such that $e_{Q}^{-1}(0)$ contains $V_{1} \times X_{2}$ for some algebraic set $X_{2}$ of codimension $\operatorname{cd}_{Q} A$ in the finite-dimensional space $V_{2}$. Let $V_{1}^{\prime}=V_{1} \cap I^{n}$, and let $V_{1}^{\prime \prime}$ denote a complementary subspace to $V_{1}^{\prime}$ in $V_{1}$. Let $V_{2}^{\prime}=V_{2} \oplus V_{1}^{\prime \prime}$ and $X_{2}^{\prime}=X_{2} \times V_{1}^{\prime \prime}$. Then $X_{2}^{\prime}$ is of codimension $\operatorname{cd}_{Q} A$ in $V_{2}^{\prime}$, and $V_{1}^{\prime} \times X_{2}^{\prime}=V_{1} \times X_{2} \subset e_{Q}^{-1}(0)$. Replacing $V_{1}, V_{2}, X_{2}$ by $V_{1}^{\prime}, V_{2}^{\prime}, X_{2}^{\prime}$, we may therefore assume that $V_{1} \subset I^{n}$.

We identify $V_{2}$ with the $k$-points of the variety $\mathbb{A}^{\operatorname{dim} V_{2}}$. Let $\underline{X}_{2}$ denote the Zariski closure of $X_{2}$ in $\mathbb{A}^{\operatorname{dim} V_{2}}$, so we can identify $X_{2}$ with $\underline{X}_{2}(k)$, and $\operatorname{dim} \underline{X}_{2}=\operatorname{dim} V_{2}-\operatorname{cd} Q A$. We identify $(A / I)^{n}$ with the $k$-points of $\mathbb{A}^{n \operatorname{dim} A / I}$ and denote by $\underline{Y}$ the Zariski closure of the algebraic set

$$
Y=\left\{\left(\bar{a}_{1}, \ldots, \bar{a}_{n}\right) \in(A / I)^{n} \mid e_{Q}\left(\bar{a}_{1}, \ldots, \bar{a}_{n}\right)=0\right\},
$$

so the algebraic set is identified with $\underline{Y}(k)$.

The projection map $A^{n} / V_{1} \rightarrow A^{n} / I^{n}$ maps the algebraic set $X_{2}$ to the algebraic set $Y$, and it follows that the associated projection morphism $\mathbb{A}^{\operatorname{dim} V_{2}} \rightarrow \mathbb{A}^{n \operatorname{dim} A / I}$ defines a morphism $\pi: \underline{X}_{2} \rightarrow \underline{Y}$. As $0 \in \underline{X}_{2}(k)=X_{2}$ maps by $\pi$ to $0 \in \underline{Y}(k)=Y$, the fiber $\underline{Z}$ of $\pi$ over 0 is non-empty. Now, $\underline{Z}(k)$ is $X_{2} \cap\left(I^{n} / V_{1}\right)$, so $\operatorname{cd}_{Q} I \leq \operatorname{dim}\left(I^{n} / V_{1}\right)-\operatorname{dim} \underline{Z}$. By [Stacks, Tag 02JS],

$$
\operatorname{dim} \underline{X}_{2} \leq \operatorname{dim} \underline{Y}+\operatorname{dim} \underline{Z} \text {. }
$$

Thus,

$$
\begin{aligned}
\operatorname{cd}_{Q} A & =\operatorname{dim} A^{n} / V_{1}-\operatorname{dim} \underline{X}_{2}=\operatorname{dim} A^{n} / I^{n}+\operatorname{dim} I^{n} / V_{1}-\operatorname{dim} \underline{X}_{2} \\
& \geq \operatorname{dim} A^{n} / I^{n}-\operatorname{dim} \underline{Y}+\operatorname{dim} I^{n} / V_{1}-\operatorname{dim} \underline{Z} \geq \operatorname{cd}_{Q}(A / I)+\operatorname{cd}_{Q} I .
\end{aligned}
$$

We can now prove Theorem 1 .

Proof. It suffices to prove that, if condition (2) does not hold, then for all $i \geq 0$ there exists an ideal $I$ of finite codimension in $A$ such that $\operatorname{cd}_{Q} A / I \geq i$. We proceed by induction on $i$, the statement being trivial for $i=0$. If the induction hypothesis holds, as $Q$ is not an identity for $I$, there exists $\alpha \in I^{n}$ with $e_{Q}(\alpha) \neq 0$. As $A$ is residually finite dimensional, there exists an ideal $J$ of $A$ of finite codimension such that $e_{Q}(\alpha) \notin J$. If $\bar{\alpha}$ denotes the image of $\alpha$ in $(I / I \cap J)^{n}$, then $e_{Q}(\bar{\alpha}) \neq 0$, so $Q$ is not an identity for $I / I \cap J$. By Proposition 4 .

$$
\operatorname{cd}_{Q} A /(I \cap J) \geq \operatorname{cd}_{Q} A / I+\operatorname{cd}_{Q} I /(I \cap J) \geq i+1,
$$

and the theorem follows by induction.

We now discuss some consequences of the theorem.

Corollary 5. Let $k$ be a field of characteristic 0, A a finitely generated algebra over $k$, and $Q$ a homogeneous polynomial in $n$ variables defined over $k$. If $V$ is a subspace of $A^{n}$ of finite codimension, $\vec{a}=\left(a_{1}, \ldots, a_{n}\right) \in A^{n}$, and $e_{Q}(\vec{a}+V)=0$, then $Q$ is an identity on an ideal of $A$ of finite codimension. 
Proof. As $A \otimes_{k} \bar{k}$ is finitely generated over $\bar{k}$, it is residually finite-dimensional. As $k$ is infinite, if $Q$ is an identity on $\vec{a}+V$, then it is an identity on $\vec{a}+V \otimes_{k} \bar{k}$, which is a subset of finite codimension in $A \otimes_{k} \bar{k}$. Therefore, there exists an ideal $I$ of finite codimension in $A \otimes_{k} \bar{k}$ such that $Q$ is an identity on $I$.

Let $\left\{a_{1}, \ldots, a_{m}\right\}$ be a generating subset for $A$ over $k$. Let $\bar{a}_{i}$ denote the image of $a_{i} \otimes 1$ under the quotient homomorphism

$$
A \otimes_{k} \bar{k} \rightarrow\left(A \otimes_{k} \bar{k}\right) / I .
$$

The $\bar{k}$-algebra $\left(A \otimes_{k} \bar{k}\right) / I$ is finite-dimensional, so fixing any basis, the structure constants lie in a finite extension $K$ of $k$. We fix a finite-dimensional $K$-algebra $B$ and an isomorphism of $K$-algebras $\iota: B \otimes_{K} \bar{k} \rightarrow\left(A \otimes_{k} \bar{k}\right) / I$. There exists a finite extension $L / K$ such that $\iota^{-1}\left(\bar{a}_{i}\right) \in B \otimes_{k} L$ for all $i$. Enlarging $L$, we may assume $L / K$ is Galois. There is a unique $L$-algebra homomorphism $\phi: A \otimes_{k} L \rightarrow B \otimes_{K} L$ such that the diagram

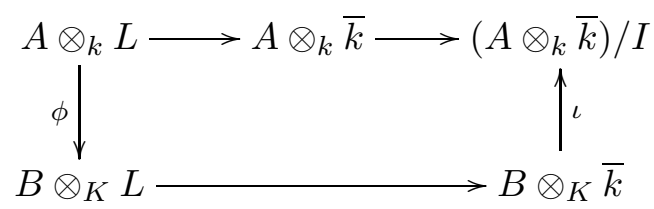

commutes.

We consider the $L$-algebra homomorphism

$$
A \otimes_{k} L \rightarrow \bigoplus_{\sigma \in \operatorname{Gal}(L / k)} B \otimes_{K} L
$$

which in the $\sigma$-coordinate is given by $\left(\operatorname{Id}_{B} \otimes \sigma\right) \circ \phi$. The kernel is invariant under the action of $\operatorname{Gal}(L / k)$ on $A \otimes_{k} L$, so by Galois descent for vector spaces [B, Chap. 5, $\S 10$ Prop. 6] it is of the form $W \otimes_{k} L$, where $W$ is the kernel of the composition of $A \rightarrow A \otimes_{k} L$ and $\phi$. Thus $W$ is a finite codimension ideal of $A$.

Corollary 6. Let $A$ be a residually finite dimensional associative algebra over $k$. Let $d \geq 1$ and suppose $x^{d}$ is an almost identity for $A$. If $k$ has characteristic $p>0$ suppose also $p>d$. Then $A$ has an ideal I of finite codimension satisfying $I^{f(d)}=0$, where $f(d)$ is a suitable function of $d$.

Proof. By Theorem 1, $A$ has an ideal $I$ of finite codimension satisfying the identity $x^{d}=0$.

The well known Nagata-Higman Theorem applied for the associative (nonunital) $k$-algebra $I$ shows that $I^{f(d)}=0$. See for instance [DF, Chapter 6] for the theorem and for explicit bounds on the function $f$.

Our next result describes almost $d$-Engel Lie-algebras.

Theorem 7. Let $L$ be a residually finite dimensional Lie algebra over $k$. Let $d \geq 1$ and suppose The Engel polynomial $E_{d}$ is an almost identity for $L$. Then 
(1) If $k$ has characteristic zero then $L$ has a nilpotent ideal of finite codimension.

(2) If $k$ has positive characteristic then $L$ has a locally nilpotent ideal of finite codimension.

Proof. By Theorem 1, $L$ has an ideal $I$ of finite codimension satisfying the identity $E_{d}=0$. The conclusion now follows from well known theorems of Zelmanov on the nilpotency of $d$-Engel Lie algebras in characteristic zero [Z1] and the local nilpotency of $d$-Engel Lie algebras in positive characteristic $[\mathrm{Z} 2, \mathrm{Z3}]$.

\section{Algebras over finite FIELdS}

From now on, we assume $k=\mathbb{F}_{q}$. For every positive integer $d$, we write $d=m(q-1)+r$, where $0 \leq r \leq q-2$, and define

$$
f_{q}(d)=\frac{q-r}{q^{m+1}}
$$

Lemma 8. We have

$$
f_{q}(d)=\min \prod_{i=1}^{\infty} \frac{q-x_{i}}{q},
$$

where the sum ranges over all infinite real sequences $x_{1}, x_{2}, x_{3}, \ldots \in[0, q-1]$ summing to $d$.

Proof. As $\log (q-x)$ is concave, the value of the product can only decrease if we replace the sequence with

$$
x_{1}, \ldots, x_{n-1}, \sum_{i=n}^{\infty} x_{i}, 0,0, \ldots
$$

Thus, we may consider only sequences which are eventually zero. If any two non-zero terms $x_{i}$ and $x_{j}$ satisfy $x_{i}+x_{j} \leq q-1$, then we can decrease the product by replacing $x_{i}$ and $x_{j}$ by $x_{i}+x_{j}$ and 0 respectively, so we may assume any two non-zero terms sum to more than $q-1$. If $q-1>x_{i}>x_{j}>0$ and $x_{i}+x_{j}>q-1$, then we can decrease the product by replacing $x_{i}$ and $x_{j}$ by $q-1$ and $x_{i}+x_{j}-(q-1)$ respectively. Thus, we may assume that there is at most one $x_{i}$ which is neither 0 nor $q-1$. Without loss of generality, the sequence can be taken to be non-increasing, so the minimum is achieved for

$$
x_{1}=\cdots=x_{m}=q-1, x_{m+1}=r, x_{m+2}=\cdots=0 .
$$

Lemma 9. For $1 \leq k \leq q-1$ and $d \geq k$,

$$
f_{q}(d) \leq \frac{q-k}{q} f_{q}(d-k) .
$$

Proof. This follows immediately from the description of $f_{q}$ in Lemma 8 ,

Lemma 10. For $q \geq 2$, we have $f_{q}(d) \geq 2^{-d}$. 
Proof. We have $f_{q}(0)=1$, and writing $d=m(q-1)+r$, with $0 \leq r \leq q-2$, we have

$$
\frac{f_{q}(d+1)}{f_{q}(d)}=\frac{q-r-1}{q-r} \geq \frac{1}{2}
$$

Theorem 11. Let $\mathbb{F}_{q}$ be a finite field and $P\left(x_{1}, \ldots, x_{n}\right) \in \mathbb{F}_{q}\left[x_{1}, \ldots, x_{n}\right] a$ polynomial of degree $d$. If

$$
N_{P}:=\left|\left\{\left(a_{1}, \ldots, a_{n}\right) \in \mathbb{F}_{q}^{n} \mid P\left(a_{1}, \ldots, a_{n}\right) \neq 0\right\}\right|
$$

is non-zero, it satisfies

$$
N_{P} \geq f_{q}(d) q^{n} \geq 2^{-d} q^{n} .
$$

Proof. As a function on $\mathbb{F}_{q}^{n}, P$ depends only on its residue class modulo $\left(x_{1}^{q}-x_{1}, \ldots, x_{n}^{q}-x_{n}\right)$. Each such residue class contains a unique element which is of degree $<q$ in each variable separately, and the (total) degree of this representative achieves the minimal degree of all polynomials in the residue class of $P$. As $f_{q}(d)$ decreases monotonically in $d$, we may assume that $P$ is of degree less than $q$ in each variable separately.

We use induction on $d$, the base case $d=0$ being trivial. Without loss of generality, we may assume that, as a function on $\mathbb{F}_{q}^{n}, P$ is not constant in the variable $x_{n}$. Since the $x_{n}$-degree of $P$ is less than $q$, this means that the $x_{n}$-degree is $l \in[1, q-1]$. We write

$$
P\left(x_{1}, \ldots, x_{n}\right)=\sum_{i=0}^{l} P_{i}\left(x_{1}, \ldots, x_{n-1}\right) x_{n}^{i} .
$$

As $l+\operatorname{deg} P_{l}=\operatorname{deg} x_{n}^{l} P_{l} \leq \operatorname{deg} P$, we have $\operatorname{deg} P_{l} \leq d-l$. If $\left(a_{1}, \ldots, a_{n-1}\right) \in$ $\mathbb{F}_{q}^{n-1}$ satisfies $P_{l}\left(a_{1}, \ldots, a_{l-1}\right) \neq 0$, there are at least $q-l$ solutions of $P\left(a_{1}, \ldots, a_{n-1}, x_{n}\right) \neq 0$. By the induction hypothesis,

$$
N_{P} \geq(q-l) N_{P_{l}} \geq(q-l) f_{q}(d-l) q^{n-1}=\frac{q-l}{q} f_{q}(d-l) q^{n} \geq f_{q}(d) q^{n} .
$$

We can now prove Theorem 3 ,

Proof. We have to show that, if the evaluation map $e_{Q}$ associated with $Q$ is not identically 0 , then $\frac{\left|e_{Q}^{-1}(0)\right|}{|A|^{n}}<1-2^{-d}$.

If $e_{Q}$ does not vanish on $A$, then there exists a linear functional $f: A \rightarrow \mathbb{F}_{q}$ such that $f \circ e_{Q}$ does not vanish on $A^{n}$. If $Q$ is of degree $d$, then $f \circ e_{Q}$ has degree $\leq d$. Theorem 3 now follows from Theorem 11 .

Let $n$ be a positive integer and consider $\mathbb{F}_{q}\left\langle x_{1}, \ldots, x_{n}\right\rangle$, the algebra over $\mathbb{F}_{q}$ of the free magma on $n$ generators. This is a graded $\mathbb{F}_{q}$-algebra. Let $Q \in \mathbb{F}_{q}\left\langle x_{1}, \ldots, x_{n}\right\rangle$ denote a non-zero element of degree $d$. If $I$ is any ideal 
of $A$ of finite codimension, then $Q$ induces a map $(A / I)^{n} \rightarrow A / I$, which we denote $Q_{I}$. Let

$$
f(Q, I):=\frac{\left|Q_{I}^{-1}(0)\right|}{|A / I|^{n}} .
$$

Regarding $A / I$ as a finite-dimensional vector space, $Q_{I}$ is given by a polynomial of degree $\leq d$, so either it maps $(A / I)^{n}$ to 0 , or

$$
f(Q, I) \leq 1-2^{-d} .
$$

We now prove Theorem 2 .

Proof. We first prove that condition (1) implies condition (2). We have to show that if $Q$ is not a coset identity of $A$ then for all $\epsilon>0$, there exists an ideal $I$ of finite codimension such that $f(Q, I) \leq \epsilon$. We first prove that it implies that for any ideal $I$ of finite codimension, there exists an ideal $J \subset I$ of finite codimension such that

$$
f(Q, J) \leq\left(1-2^{-d}\right) f(Q, I) .
$$

For each element $\alpha \in Q_{I}^{-1}(0)$, we choose a representative $\tilde{\alpha}=\left(a_{1}, \ldots, a_{n}\right) \in$ $A^{n}$ such that $e_{Q}\left(a_{1}, \ldots, a_{n}\right) \neq 0$ and an ideal of finite codimension $I_{\alpha}$ to which $e_{Q}\left(a_{1}, \ldots, a_{n}\right) \neq 0$ does not belong. Let

$$
J=I \cap \bigcap_{\alpha} I_{\alpha}
$$

which, by construction, is of finite codimension. Again by construction, $Q_{J}$ does not map any $n$-tuple of cosets of $I / J$ to 0 . Therefore, for each such $n$ tuple, the number of elements mapping to 0 by $Q_{J}$ is at most $\left(1-2^{-d}\right)|I / J|^{n}$. If the coset maps to an element of $(A / I)^{n}$ which is not in $Q_{I}^{-1}(0)$, then no element of that coset maps to 0 by $Q_{J}$. This proves the claim, and the equivalence of conditions (1) and (2) follows immediately.

Now, suppose $Q$ is multilinear. We will show that condition (2) implies condition (3) with the same ideal $I$. Assuming (2) we have

$$
e_{Q}\left(a_{1}+y_{1}, \ldots, a_{n}+y_{n}\right)=0
$$

for all $y_{1}, \ldots, y_{n} \in I$. By the multilinearity of $Q$ we have, for all $y_{1} \in I$,

$$
\begin{aligned}
0=e_{Q}\left(a_{1}+y_{1}, a_{2}, \ldots, a_{n}\right) & =e_{Q}\left(a_{1}, a_{2}, \ldots, a_{n}\right)+e_{Q}\left(y_{1}, a_{2}, \ldots, a_{n}\right) \\
& =e_{Q}\left(y_{1}, a_{2}, \ldots, a_{n}\right) .
\end{aligned}
$$

Similarly we have, for all $y_{2} \in I$,

$0=e_{Q}\left(y_{1}, a_{2}+y_{2}, a_{3}, \ldots, a_{n}\right)=e_{Q}\left(y_{1}, a_{2}, a_{3}, \ldots, a_{n}\right)+e_{Q}\left(y_{1}, y_{2}, a_{3}, \ldots, a_{n}\right)$.

Proceeding in this way we obtain

$$
e_{Q}\left(y_{1}, \ldots, y_{n}\right)=0
$$

for all $y_{1}, \ldots, y_{n} \in I$.

This completes the proof. 


\section{REFERENCES}

[A] Amitsur, Shimshon: An embedding of PI-rings, Proc. Amer. Math. Soc. 3 (1952), 3-9.

[B] Bourbaki, Nicolas: Éléments de mathématique. Algèbre. Chapitres 4 à 7 . Masson, Paris, 1981.

[D] Dixon, John: Probabilistic group theory, C.R. Math. Acad. Sci. Soc. R. Can. 24 (2002), 1-15.

[DF] Drensky, Vesselin; Formanek, Edward: Polynomial identity rings. Advanced Courses in Mathematics, CRM Barcelona, Birkhäuser Verlag, Basel, 2004.

[LS] Larsen, Michael; Shalev, Aner: Almost PI algebras are PI, arXiv::1910.05764, to appear Proc. Amer. Math. Soc.

[MM] Mann, Avinoam; Martinez, Consuelo: Groups nearly of prime exponent and nearly Engel Lie algebras, Arch. Math. (Basel) 71 (1998), no. 1, 5-11.

[Stacks] Stacks Project, https://stacks.math.columbia.edu.

[Z1] Zelmanov, Efim: Engel Lie algebras. (Russian) Dokl. Akad. Nauk SSSR 292 (1987), no. 2, 265-268.

[Z2] Zelmanov, Efim: Solution of the restricted Burnside problem for groups of odd exponent, (Russian) Izv. Akad. SSSR Ser. Mat. 54 (1990), 42-59, 1990; translation in Math USSR-Izv. 36 (1990), 41-60.

[Z3] Zelmanov, Efim: Solution of the restricted Burnside problem for 2-groups, (Russian) Mat. Sb. 182 (1991), 568-592; translation in Math USSR-Sb 72 (1992), 543-565.

E-mail address: mjlarsen@indiana.edu

Department of Mathematics, Indiana University, Bloomington, IN 47405, U.S.A.

E-mail address: shalev@math.huji.ac.il

Einstein Institute of Mathematics, Hebrew University, Givat Ram, Jerusalem 91904, ISRAEL 